\title{
Datos, modelos y morfismos : sobre el estructuralismo intuitivo de Suppes
}

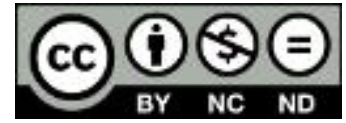

Esta obra está bajo una Licencia Creative Commons Argentina.

Atribución - No Comercial - Sin Obra Derivada 2.5

https://creativecommons.org/licenses/by-nc-nd/2.5/ar/

Documento descargado de RIDAA-UNQ Repositorio Institucional Digital de Acceso Abierto de la Universidad Nacional de Quilmes de la Universidad Nacional de Quilmes

\section{Cita recomendada:}

Rolleri, J. L. (2014). Datos, modelos y morfismos: sobre el estructuralismo intuitivo de Suppes. Metatheoria, 4(2), 1-8. Disponible en RIDAA-UNQ Repositorio Institucional Digital de Acceso Abierto de la Universidad Nacional de Quilmes http://ridaa.unq.edu.ar/handle/20.500.11807/2436 


\title{
Datos, modelos y morfismos: sobre el estructuralismo intuitivo de Suppes
}

Data, Models and Morphisms: On Suppes's Intuitive Structuralism

José Luis Rolleri ${ }^{\dagger}$

\begin{abstract}
Resumen
En este escrito se analizan ciertos conceptos del estructuralismo intuitivo de Patrick Suppes, en particular, el de modelo de datos, para dar una réplica a la crítica de Muller a Suppes de que la axiomatización conjuntista de una teoría física conlleva la pérdida de su contenido empírico. Para ello, antes, intento una mejora formal a la propuesta de Suppes sobre cómo se conectan los modelos de los experimentos con los modelos teóricos.
\end{abstract}

Palabras clave: modelos de datos - subestructura parcial - interpretación intencional - contenido empírico

\begin{abstract}
In this paper I analyze certain concepts of Patrick Suppes' intuitive structuralism, in particular that of model of data, in order to give a reply to Muller's critique to Suppes that the set-theoretical axiomatization of a physical theory involves the loss of its empirical content. With that intention, before, I try a formal improvement of Suppes' propose on how the models of experiments are connected with the theoretical models.
\end{abstract}

Keywords: models of data - partial substructure - intended interpretation - empirical content

\footnotetext{
${ }^{*}$ Recibido: 3 de Noviembre de 2013. Aceptado en versión revisada: 27 de Febrero de 2014.

† Universidad Autónoma de Querétaro, México. Para contactar al autor, por favor escriba a: jlrolleri@yahoo.com. Metatheoria 4(2)(2014): 1-8. ISSN 1853-2322.

(C) Editorial de la Universidad Nacional de Tres de Febrero. Publicado en la República Argentina.
} 


\section{Introducción}

¿Cuál es la relación que guarda una teoría física con los experimentos? Ésta es una de las cuestiones centrales de la filosofía de la ciencia. Adopta una formulación más específica cuando se pregunta sobre la conexión entre las predicciones teóricas y los datos experimentales. La línea de respuesta que, en general, recibe esta cuestión es, a grandes rasgos, que los datos que se obtienen de observaciones, experimentos y mediciones deben ajustarse -salvo margen de error- con los valores numéricos o la expresión cuantitativa de la predicción que se derive de la teoría física. Desde la concepción semántica o modelística de las teorías, actualmente dominante, podemos preguntar, ¿cómo es que los datos de un experimento pueden ajustarse con (las predicciones de) un modelo de una teoría de la física?

En este escrito se analizan algunos conceptos claves del estructuralismo intuitivo de Patrick Suppes, como recientemente lo ha llamado Fred Muller (2011), para dar una réplica a la crítica de Muller a Suppes con respecto a la cuestión de que las teorías físicas pierden su contenido empírico o factual cuando son axiomatizadas conjuntistamente. Para ello antes intentaré ofrecer una mejora formal a la propuesta de Suppes sobre cómo se conectan los modelos de datos con los modelos de una teoría física y, de ahí, sobre cómo la experiencia experimental está relacionada con la teoría.

\section{La propuesta de Suppes}

El concepto de modelo de datos fue propuesto por Patrick Suppes para "tratar de mostrar que el análisis exacto de la relación entre teorías empíricas y datos relevantes precisa de una jerarquía de modelos de diferente tipo lógico" (Suppes 1988b, p. 148). Según Suppes, en la cúspide de la jerarquía de modelos correspondiente a una teoría empírica estarían los modelos de la teoría misma; en cada uno de los niveles inferiores habría una teoría por derecho propio -que corresponderían a las teorías y modelos de medición fundamental y derivada de las magnitudes involucradas-, y en la base los modelos de datos que tendrían que estar vinculados con la experiencia experimental misma. Además, la teoría en cada nivel adquiriría significado empírico por medio de conexiones formales con la teoría en un nivel más bajo (ver Suppes 1988b, p. 159). ${ }^{1}$

El concepto de modelo que Suppes emplea es el lógico; esto es: "[...] un modelo de una teoría puede definirse como una realización posible en la cual se satisfacen todos los enunciados válidos de la teoría" (Suppes 1988b, p. 147), donde una realización posible es una entidad con una estructura conjuntista apropiada. Con respecto a los datos experimentales, este concepto involucra que hay una teoría del experimento que esos datos deben satisfacer. Sobre ello, Suppes sostiene que "La definición precisa de los modelos de los datos para cualquier experimento dado, requiere que haya una teoría de los datos en el sentido del procedimiento experimental, así como en el sentido ordinario de la teoría empírica de los fenómenos que están siendo estudiados" (Suppes 1988b, p. 149).

La postulación de Suppes de jerarquías completas de modelos parece una idealización de lo que uno puede esperar encontrar o una versión de casos ideales. Específicamente, los bloques para construir la base de ese género de jerarquías que Suppes propone, son: 1) una descripción del procedimiento experimental, incluido el aparato usado; 2) los datos brutos que arrojan los ensayos del experimento; 3) la sistematización de estos datos en una estructura conjuntista apropiada, como una realización posible de la tácita teoría del experimento, y; 4) la extracción de las frecuencias relativas con que ocurren los datos relevantes de esas realizaciones posibles.

Las anteriores estructuras conjuntistas de datos referidas en (3) como realizaciones posibles, cumplen el papel de proveer de la información relevante obtenida del experimento. Además, como el

\footnotetext{
${ }^{1}$ El carácter empírico y operacional que Suppes y sus colegas le atribuyen a las teorías de la medición fundamental de magnitudes, como la longitud, ha sido convincentemente criticado por Batitsky (1998), mostrando que, incluso, a ese nivel básico de construcción de modelos de medición se precisa de varias idealizaciones. La teoría estructuralista de la medición de Balzer no otorga prioridad a la observación directa ni a formas distinguidas de medición, como la llamada 'fundamental' (cfr. Balzer 1992, p. 108).
} 
propio Suppes anota en otro lugar, las frecuencias relativas extraídas de las realizaciones posibles pueden compararse con los enunciados probabilistas derivados de la teoría básica, o en sus propias palabras: "Los datos de esos ensayos se analizan luego en términos de la comparación de los mismos entre las frecuencias relativas observadas de los datos y las probabilidades predichas por la teoría" (Suppes 1988c, p. 135).

Sin embargo, un análisis comparativo entre una estructura de datos y un modelo afronta dificultades, como es el caso de que en las teorías físicas hay nociones que no tienen un análogo directo observable en los datos experimentales, y de que los modelos de una teoría contienen funciones continuas y secuencias infinitas, mientras que los datos experimentales son de carácter discreto y altamente finitos (cfr. Suppes 1988b pp. 148-149).

Es claro que surgen dificultades desde el momento que uno quiere comparar las estructuras de los modelos de datos y las estructuras de los modelos de las teorías, empezando con el hecho que, en general, serían estructuras de distinto tipo. En general, como lo indica la anterior observación de Suppes, los modelos de las teorías físicas tienen una estructura más compleja que la estructura de los modelos de datos, además de contener secuencias infinitas y funciones definidas en conjuntos continuos. Las funciones llamadas 'homomorfismo' e 'isomorfismo' requieren que las dos estructuras en las que se definen, sean del mismo tipo. Esto último significa una severa restricción, puesto que difícilmente se tendría que un modelo de datos sea del mismo tipo que un modelo teórico.

Para apreciar las dificultades involucradas en la tesis de que hay conexiones formales entre los modelos de datos y los modelos teóricos es conveniente definir unas nociones. Iniciaremos con la noción de estructura relacional. ${ }^{2}$

D1. $\mathfrak{C}$ es una estructura si y sólo si existen $D_{1}, \ldots, D_{k}$ y $f_{1}, \ldots, f_{m}$ tales que

(1) $\mathfrak{C}=\left\langle D_{1}, \ldots, D_{k} ; f_{1}, \ldots, f_{m}\right\rangle D_{1}, \ldots, D_{k}$ son conjuntos no-vacíos

(2) para todo $i \leq m$ se cumple que $f_{i}$ es una relación sobre $D_{1}, \ldots, D_{k}$.

D2. Sean $D_{1}, \ldots, D_{k}$ y $D_{1}^{\prime}, \ldots, D_{n}^{\prime}$ conjuntos, con $k=n$. Sean además $f$ una relación sobre $D_{1}, \ldots, D_{k}$ y $f^{\prime}$ una relación sobre $D_{1}^{\prime}, \ldots, D_{n}^{\prime}$. Entonces $f$ y $f^{\prime}$ son de idéntico tipo si y sólo si hay $i_{1}, . ., i_{m} \in$ $\{1, \ldots, k\}$ tales que

$f \subseteq \prod D_{i j}$ y $f^{\prime} \subseteq \prod D_{i j}^{\prime}$, con $j \leq m$, donde

$\prod D_{i j}=D_{1} \times \ldots \times D_{j}$.

D3. Sean $\mathfrak{C}=\left\langle D_{1}, \ldots, D_{k} ; f_{1}, \ldots, f_{m}\right\rangle$ y $\mathfrak{C}^{\prime}=\left\langle D_{1}^{\prime}, \ldots, D_{n}^{\prime} ; f_{1}^{\prime}, \ldots, f_{s}^{\prime}\right\rangle$ dos estructuras. Entonces $\mathfrak{C}_{\text {y }} \mathfrak{C}^{\prime}$ son de idéntico tipo si y sólo si

(1) $k=n y m=s$

(2) para toda $i \leq m$ se cumple que $f_{i}$ es de idéntico tipo que $f_{i}^{\prime}$.

D4. Sean $\mathfrak{C}=\left\langle D_{1}, \ldots, D_{k} ; f_{1}, \ldots, f_{n}\right\rangle$ y $\mathfrak{C}^{\prime}=\left\langle D^{\prime}{ }_{1}, \ldots, D_{k}^{\prime} ; f_{1}^{\prime}, \ldots, f_{n}^{\prime}\right\rangle$ dos estructuras de idéntico tipo.

Entonces $h$ es un homomorfismo de $\mathfrak{C}$ en $\mathfrak{C}^{\prime}$ si y sólo si

(1) para todo $i \leq k, h$ es una función inyectiva de $D_{i}$ en $D_{i}^{\prime}$

(2) para todo $j \leq n$, si $f_{j}\left(x_{1} \ldots x_{m}\right)$ entonces $f_{j}^{\prime}\left(h\left(x_{1}\right) \ldots h\left(x_{m}\right)\right)$.

D5. Sean $\mathbb{C}=\left\langle D_{1}, \ldots, D_{k} ; f_{1}, \ldots, f_{n}\right\rangle$ y $\mathbb{C}^{\prime}=\left\langle D_{1}^{\prime}, \ldots, D_{k}^{\prime} ; f_{1}^{\prime}, \ldots, f_{n}^{\prime}\right\rangle$ dos estructuras de idéntico tipo.

Entonces $g$ es un isomorfismo de $\mathfrak{C}$ sobre $\mathfrak{C}$ ' si y sólo si

(1) para todo $i \leq k, g$ es una función biyectiva de $D_{i}$ sobre $D_{i}^{\prime}$

(2) para todo $j \leq n, f_{j}\left(x_{1} \ldots x_{m}\right)$ si y sólo si $f_{j}^{\prime}\left(g\left(x_{1}\right) \ldots g\left(x_{m}\right)\right)$.

Como puede observarse, la relación de isomorfismo entre estructuras es una relación sumamente fuerte, que suele cumplirse sólo en estructuras que son modelos de una misma teoría. Intuitivamente hablando, expresa la idea de que dos modelos tienen exactamente la misma forma y difieren un tanto

2 Véase Balzer (1997), pp. 279-281 y 285-286, para las definiciones D1-D3 y D6. 
en su contenido. Nótese que esa relación precisa que los dominios respectivos de las dos estructuras sean de la misma cardinalidad y que toda relación en las estructuras se preserve en ambos sentidos. Por un lado, el o los dominios de las estructuras de datos, en general, podrían tener una cardinalidad menor que el o los dominios del modelo teórico. Por otro lado, como Suppes anota, están los casos en los que los modelos de la teoría física contienen conceptos (magnitudes) que no tienen su contraparte en la estructura de datos. En cualquiera de ambos casos, no se podría definir una función isomórfica.

La noción de homomorfismo, aunque es más débil que la de isomorfismo, resulta demasiado exigente porque requiere también que las estructuras sean de idéntico tipo. En términos intuitivos, expresa la idea de que las estructuras de dos modelos son tan similares, difiriendo en contenido, que una se puede subsumir en la otra, preservando las relaciones. En este contexto, esto implica que en la estructura de datos hay un dominio correspondiente a cada dominio en el modelo teórico, y que a cada concepto (magnitud) del modelo de la teoría hay en la estructura de datos una relación que le corresponde. Igualmente, ambas condiciones resultan implausibles de ser cumplidas, puesto que, generalmente, el modelo teórico será más rico y complejo en estructura que alguna estructura de datos.

Si bien hay lugar a hacer ciertas idealizaciones y abstracciones, para poder subsumir una estructura de datos en un modelo teórico, en general definir la función que se precisaría para ello será un tanto implausible.

Para buscar alternativas, introducimos unas nociones más débiles de estructuras.

D6. Sean $\mathfrak{C}=\left\langle D_{1}, \ldots, D_{k} ; f_{1}, \ldots, f_{n}\right\rangle$ y $\mathfrak{C}^{\prime}=\left\langle D_{1}^{\prime}, \ldots, D_{k}^{\prime} ; f_{1}{ }^{\prime}, \ldots, f_{n}\right\rangle$ dos estructuras. Entonces $\mathfrak{C}^{\prime}$ es una subestructura de $\mathfrak{C}, \mathfrak{C}^{\prime} \subseteq \mathfrak{C}$, si y sólo si

(1) para todo $i \leq k$ se cumple que $D_{i}^{\prime} \subseteq D_{i}$

(2) para todo $j \leq n$ se cumple que $f_{j}^{\prime} \subseteq f_{j}$.

Un caso especial de la anterior noción es cuando la subestructura es parcial. ${ }^{3}$

D7. Sea que $\mathfrak{C} \mathfrak{C} \subseteq \mathfrak{C}$. Entonces $\mathfrak{C}^{\prime}$ es una subestructura parcial de $\mathfrak{C}$ si y sólo si para alguna $f$ en $\mathfrak{C}$ no hay una $f^{\prime}$ en $\mathbb{C}^{\prime}$ tal que $f^{\prime} \subseteq f$.

Este par de nociones de subestructura hacen más plausible la comparación entre estructuras de datos y modelos teóricos, porque permiten que la restricción $\mathfrak{C}^{\prime}$ sea de diferente tipo que $\mathfrak{C}$. Una subestructura puede contener conjuntos vacíos como componentes. Esto es una consecuencia de D6.1 porque el conjunto vacío es subconjunto de cualquier conjunto. Además, y esto es de suma importancia, la subestructura de una realización posible infinita puede ser finita o, mejor, la restricción de un dominio infinito de una realización posible $\mathfrak{C}$ en una subestructura $\mathfrak{C}$ ' puede ser a un dominio finito. La importancia de esto se debe a que toda estructura de datos, por definición y de hecho, es finita.

Por su parte, la noción de subestructura parcial hace explícita la posibilidad de que una subestructura $\mathfrak{C}^{\prime}$ carezca de la relación correspondiente a una relación en $\mathfrak{C}$. Esta noción permite eliminar en una estructura $\mathfrak{C}$ alguna relación o, equivalentemente, sustituirla por el conjunto vacío, que correspondiera a un concepto (magnitud) en el modelo de la teoría física.

Como veremos adelante, esta última noción de subestructura parcial de una realización posible resulta la más plausible para adecuarse a alguna tesis sobre cómo se ajustan los datos de experimentos, observaciones o mediciones con los modelos de una teoría. Desde un punto de vista metodológico, es mejor considerar a las realizaciones posibles de una teoría física -las estructuras candidatas a modelosque a los modelos, porque así no se asume ni se afirma que esas estructuras relacionales cumplan con los axiomas. Desde un punto de vista formal, esto no afecta porque unas y otros son de idéntico tipo.

Ahora bien, la estrategia consiste en obtener subestructuras o, mejor, subestructuras parciales de las realizaciones posibles de una teoría, para compararlas con las estructuras de datos, restringiendo a las realizaciones posibles a ciertas subestructuras que 1) contengan conjuntos finitos, 2) contengan dominios restringidos o, incluso, se elimine algún dominio, y 3) contengan relaciones restringidas, o

\footnotetext{
${ }^{3}$ Esta noción es mencionada en Moulines (2011).
} 
bien se suprima alguna relación. De esta manera, podemos proceder a obtener subestructuras de los candidatos a modelos de una teoría que pudieran ser comparables con las estructuras finitas de datos, abriendo la posibilidad de definir homomorfismos o, quizá, isomorfismos entre ellas. Las funciones homomórficas o isomórficas se definirían, entonces, entre las estructuras finitas de datos y alguna subestructura parcial de una realización posible de la teoría física.

\section{La crítica de Muller}

Recordando que para Suppes los modelos de datos estarían vinculados directamente con la experiencia experimental y serían, a su vez, las estructuras que dotarían de contenido empírico a los modelos del siguiente nivel superior por medio de una conexión formal, preguntamos: ¿qué género de vínculo formal puede darse entre una estructura de datos y algún modelo que cumpla ese cometido, que dote de contenido empírico al modelo?

Fred Muller, en una reconstrucción de la concepción modelística de Suppes, con respecto a esto escribe:

(e) Sea $D_{t}(\mathrm{~T})$ el conjunto de estructuras de datos que se han obtenido hasta un tiempo histórico $t$ de los resultados de mediciones en los experimentos u observaciones relevantes para $\mathrm{T}$, los cuales son extraídos del 'fenómeno' que se supone $\mathrm{T}$ salve. Llamemos a $T$ [la extensión del predicado conjuntista que define a la teoría $\mathbf{T}$ ] observacionalmente adecuada en $t$ si y sólo si para cada estructura $\mathfrak{D} \in D_{t}(\mathrm{~T})$, existe alguna estructura (modelo) $\mathfrak{S} \in T$ tal que $\mathfrak{D}$ es subsumible [embeddable] en $\mathfrak{S}$, donde la 'subsumibilidad' es construida ampliamente como algún morfismo de $\mathfrak{D}$ en (alguna parte de) $\mathfrak{S}$. (Muller 2011, p. 92)

La noción apropiada para afirmar que una estructura es subsumible en un modelo es la de homomorfismo. Hemos visto que esta relación es demasiado exigente a este propósito, porque involucra que ambas estructuras sean de idéntico tipo. Difícilmente podríamos atribuirle a Suppes tal tesis, ya que él mismo anota las diferencias en las estructuras de los modelos de datos y los modelos teóricos. Sin embargo, podemos formular alguna noción apropiada -sugerida al final de la anterior cita de Muller- de subsunción parcial para expresar, en términos generales, el tipo de conexión formal entre esos géneros de modelos.

D8. Sean $E$ una estructura finita de datos y $R$ una realización posible de una teoría. Entonces $E$ es parcialmente subsumible en $R$ si y sólo si

(1) hay una subestructura $S$ de $R$ tal que $S$ contenga un dominio finito $D^{\prime}$ que corresponda a un dominio $D$ en $E$.

(2) hay una función $h$ de $E$ en $S$ tal que $h$ mapea $D$ en $D^{\prime}$ y para toda relación $f$ sobre $D$ en $E$, le corresponda una relación $f^{\prime}$ sobre $D^{\prime}$ en $S$, tal que para toda $x$, y en $D$, si $f(x, y)$ entonces $f(h(x), h(y))$.

La anterior no es sino la relación de homomorfismo $h$ formulada para estructuras finitas de datos $E$ y subestructuras $S$ de realizaciones posibles $R$ de una teoría en tanto estructuras relacionales. En el caso en que sea posible definir un homomorfismo tal, podemos decir que $E$ es subsumible en $S$ o parcialmente subsumible en $R$ y, de ahí, en un modelo de la teoría (esto no excluye la posibilidad de una subsunción no parcial en $R$ porque, por definición, toda estructura es una subestructura de sí misma).

De seguro se requerirán ciertas idealizaciones en el anterior procedimiento, tanto en la construcción de la estructura de datos como en la obtención de una subestructura parcial apropiada (que no sea ad hoc) para definir un homomorfismo. No obstante, los homomorfismos y, quizá, los isomorfismos expresarían las conexiones formales entre los modelos de datos y los modelos de una teoría, por medio de las cuales se conferiría de un contenido empírico a estos últimos modelos.

Sin embargo, para Muller esta respuesta, en términos de estructuras conjuntistas y morfismos, no sería suficiente. Él elabora un conjunto de 10 pares ordenados de números racionales como una 
estructura de datos, con el fin de mostrar que tomados como resultados experimentales podrían resultar relevantes para modelos de cuatro teorías diferentes: la óptica de rayos, la teoría de los circuitos eléctricos, la teoría cinemática de Galileo y la mecánica de partículas de Newton. Muller arguye que para decidir sobre cuál de esas teorías esa estructura de datos es relevante en un caso dado, se requiere ir más allá del enfoque conjuntista. Se requiere del lenguaje: emplear palabras que expresan conceptos y oraciones que expresan proposiciones para describir, al menos, el contexto experimental (cfr. Muller 2011, p. 101). Esto último apunta a la solución, según Muller, de un problema que generan las axiomatizaciones conjuntistas de las teorías científicas por arrojar estructuras puramente abstractas, que él llama 'el problema de la pérdida de contenido'.

De acuerdo, en términos puramente conjuntistas no puede decidirse sobre la relevancia de una estructura de datos para algún modelo teórico; los datos numéricos volcados en una estructura tal precisan de ser interpretados recurriendo al lenguaje. Sin embargo, Muller pasa por alto una cuestión relacionada que no es de menor importancia en la propuesta de Suppes. En las axiomatizaciones conjuntistas, ciertamente los axiomas, tanto estructurales como propios, se formulan puramente en términos de la teoría de conjuntos y de otras teorías matemáticas, como acertadamente señala Muller, pero el predicado mismo que se define se expresa en términos lingüísticos. En el caso de la mecánica newtoniana se define el predicado: $\Gamma=\langle P, T, s, m, f\rangle$ es un sistema de la mecánica clásica de partículas, junto con la interpretación física intencional:

\begin{abstract}
Podemos axiomatizar la mecánica clásica de partículas en términos de las cinco nociones primitivas de un conjunto $P$ de particulas, un intervalo $T$ de números reales correspondiente al tiempo transcurrido, una función posición $s$ definida en el producto cartesiano del conjunto de las partículas y el intervalo temporal, una función masa $m$ definida sobre un conjunto de partículas y una función fuerza $f$ definida sobre el producto cartesiano del conjunto de las partículas, el intervalo temporal y el conjunto de los enteros positivos. (Suppes 1988a, p. 113; cursivas agregadas).

Como estos términos expresan conceptos propios de la mecánica newtoniana, la axiomatización de esa teoría física no es puramente conjuntista ni puramente extensional. Lo anterior es suficiente, según Suppes, para especificar los modelos físicos de esa teoría: "Un modelo de la mecánica clásica de partículas es una quíntupla ordenada tal. Es suficientemente simple ver cómo un modelo físico real en el sentido de los físicos está relacionado a este sentido conjuntista de modelo. Simplemente podemos tomar el conjunto de partículas como, en el caso del sistema solar, el conjunto de los cuerpos planetarios" (Suppes 1988a, p. 114).
\end{abstract}

Ese procedimiento de especificar el significado intencional de los términos que ocurren en los axiomas de una teoría es un procedimiento estándar en las matemáticas. La diferencia reside, desde luego, en que en las teorías físicas los términos pretenden referir a objetos del mundo físico y a sus propiedades cuantitativas, mientras que los axiomas propios pretenden expresar, una vez interpretados, leyes de la teoría que prescriben los procesos que pueden sufrir los sistemas físicos.

Puede constatarse que Muller pasa por alto este recurso de explicitar la interpretación intencional que acompaña a la axiomatización conjuntista de una teoría de la física, en el hecho de que cuando él expone la concepción de Suppes deja en blanco la cláusula (d), esto es, se lee: "(d) —." (Muller 2011, p. 92). Anteriormente, al exponer la concepción lingüística recibida -elaborada, entre otros, por los empiristas lógicos- en la correspondiente cláusula (D), Muller anota la conocida dicotomía del lenguaje científico en conceptos teóricos y observacionales, y la tesis de la interpretación parcial de los primeros en términos de los segundos vía reglas de correspondencia (cfr. Muller 2011, p. 90). Lo que Muller debió escribir en (d) es algo así como: agregar o explicitar la interpretación física intencional de los conceptos que ocurren en la axiomatización.

Si bien Suppes no asume esa dicotomía empirista del lenguaje de la ciencia, mantiene la tesis acerca de que hay interpretaciones intencionales de los conceptos y las leyes por parte de los científicos -que las axiomatizaciones de las teorías deben hacer explícitas-, la cual es paralela a la tesis de Carnap de la interpretación parcial de las teorías axiomatizadas en la lógica elemental en un lenguaje observacional (véase Carnap 1956). Debido a esta omisión, Muller afirma erróneamente que la concepción suppesiana permanece en silencio con respecto a la relación entre las teorías axiomatizadas y el mundo 
físico (cfr. Muller 2011, p. 97). No obstante, en la respuesta de Suppes a Muller tácitamente acepta las críticas, anotando que él está de acuerdo, con mucho, de lo que dice Muller; en particular, no refuta las críticas con respecto al aludido problema de la pérdida de contenido ni al problema asociado de 'la pérdida de la realidad'.

\section{Conclusión}

Para concluir enfatizamos que, en conformidad con el estructuralismo intuitivo de Suppes, si en un candidato a modelo de una teoría se toma su dominio principal como un conjunto de objetos físicos en su ejemplo, P como el conjunto de los cuerpos planetarios-, si en la obtención de una subestructura parcial de esa realización posible se preserva ese dominio físico y si una estructura de datos relevante contiene ese mismo conjunto como su dominio principal, entonces resulta plausible definir un homomorfismo de la estructura de datos en la subestructura parcial del modelo, donde las relaciones $\mathrm{f}$ involucradas refieran a las magnitudes de los objetos en ese dominio común. Definir tales homomorfismos sería equivalente a mostrar que los resultados experimentales se ajustan con la teoría, expresando en términos generales la relación de concordancia entre teoría y experimento.

Conjuntamente, la estructura de datos, la subestructura de la realización posible y el homomorfismo entre ellas contribuyen a proveer de contenido empírico al modelo físicamente interpretado correspondiente, si es que la realización posible entera cumple con los axiomas propios. De esta manera, versus Muller, no hay pérdida de contenido de las teorías físicas en las axiomatizaciones conjuntistas propuestas por Suppes.

\section{Bibliografía}

Balzer, W. (1997), Teorías empiricas: modelos, estructuras y ejemplos, Madrid: Alianza. (Edición revisada por el autor y traducción castellana de Agustín González Ruiz de: Empirische Theorien: Modelle-Strukturen-Beispiele, Braunschweig: Vieweg, 1982.)

Balzer, W. (1992), “The Structuralist View of Measurement: An Extension of Received Measurement Theories”, en Savage, C.W. y P. Ehrlich (eds.), Philosophical and Foundational Issues in Measurement Theory, Londres: Lawrence Erlbaum, pp. 93-117.

Batitsky, V. (1998), “Empiricism and the Myth of Fundamental Measurement”, Synthese 116(1): 51-73.

Carnap, R. (1956), “The Methodological Character of Theoretical Concepts”, en Feigl, H. y M. Scriven (eds.), Minnesota Studies in the Philosophy of Science, Vol. 1, Minneapolis: University of Minnesota Press, pp. 38-76.

Krantz, D.H., Luce, R.D., Suppes P. y A. Tversky (1971), Foundations of Measurement, Vol. 1, Nueva York: Academic Press.

Moulines, C.U. (2011), “Cuatro tipos de desarrollo teórico en las ciencias empíricas”, Metatheoria 1(2): 11-27.

Muller, F.A. (2011), "Reflections on the Revolution at Stanford”, Synthese 183(1): 87-114.

Suppes, P. (1988a), "Una comparación del significado y los usos de los modelos en las matemáticas y las ciencias empíricas”, en Suppes, P., Estudios de filosofía y metodología de la ciencia, Madrid: Alianza, pp. 109-123. (Traducción castellana de Mario Alberto Cortez Rodríguez de: "A Comparison of the Meaning and Uses of Models in Mathematics and the Empirical Sciences", Synthese 12 (1960): 287-301.)

Suppes, P. (1988b), "Modelos de datos”, en Suppes, P., Estudios de filosofía y metodología de la ciencia, Madrid: Alianza, pp. 147-159. (Traducción castellana de Mario Alberto Cortez Rodríguez de: "Models of Data", en Nagel, E., Suppes, P. y A. Tarski (eds.), Logic, Methodology and Philosophy of Science: Proceedings of the 1960 International Congress, Stanford: Stanford University Press, 1962, pp. 252-261.)

Suppes, P. (1988c), "La estructura de las teorías y el análisis de datos”, en Suppes, P., Estudios de filosofía y metodología de la ciencia, Madrid: Alianza, pp. 125-145. (Traducción castellana de Pilar Castrillo y Eloy Rada de: "The Structure 
8| José Luis Rolleri

of Theories and the Analysis of Data", en Suppe, F. (ed.), The Structure of Scientific Theories, Urbana, Ill: University of Illinois Press, 1974, pp. 266-283.)

Suppes, P. (2011), "Future Development of Scientific Structures Closer to Experiments: Response to F.A. Muller", Synthese 183(1): 115-126. 